\title{
Lightening the Load of Document Smoothing for Better Language Modeling Retrieval
}

\author{
Mark D. Smucker and James Allan \\ Center for Intelligent Information Retrieval \\ Department of Computer Science \\ University of Massachusetts Amherst
}

\begin{abstract}
We hypothesized that language modeling retrieval would improve if we reduced the need for document smoothing to provide an inverse document frequency (IDF) like effect. We created inverse collection frequency (ICF) weighted query models as a tool to partially separate the IDF-like role from document smoothing. Compared to maximum likelihood estimated (MLE) queries, the ICF weighted queries achieved a $6.4 \%$ improvement in mean average precision on description queries. The ICF weighted queries performed better with less document smoothing than that required by MLE queries. Language modeling retrieval may benefit from a means to separately incorporate an IDF-like behavior outside of document smoothing.
\end{abstract}

Categories and Subject Descriptors: H.3.3 [Information Storage and Retrieval]: Information Search and Retrieval

General Terms: Algorithms, Experimentation

Keywords: Inverse Document Frequency, IDF, Document Smoothing, Language Modeling

\section{INTRODUCTION}

The language modeling approach to information retrieval represents queries and documents as probabilistic models [1]. While inverse document frequency (IDF) is common to many retrieval methods, it is not explicitly a part of the language modeling approach to retrieval. Zhai and Lafferty showed that language modeling contains an IDF-like like component when documents are smoothed with the collection model [2]. The IDF-like behavior is provided in the form of the inverse collection frequency (ICF). Zhai and Lafferty developed two-stage smoothing to separately control and leverage smoothing's estimation and query modeling capabilities [3]. Two-stage smoothing also eliminates the need for parameter tuning with training data. The query modeling role of smoothing describes the need for more smoothing to increase the IDF-like effect for verbose queries. Two-stage

Copyright is held by the author/owner(s).

SIGIR'06, August 6-11, 2006, Seattle, Washington, USA.

ACM 1-59593-369-7/06/0008. smoothing is competitive with, and sometimes outperforms, the best performance obtainable from Dirichlet prior and Jelinek-Mercer smoothing [3].

We hypothesize that providing the inverse collection frequency outside of document smoothing will result in improved retrieval. In a sense, removing a role from document smoothing will allow smoothing to perform better in its remaining roles.

To test our hypothesis, we weight our query models in proportion to the ICF. While this is an ad-hoc method to determine the probabilities of a query model, it does allow for the ICF to be partially separated from document smoothing.

\section{METHODS}

Maximum likelihood estimation (MLE) is a common technique for estimating query models. MLE estimates the probability of a word given a text as the count of that word divided by the total number of words in the text. As such, the MLE probability of a word $w$ given a query $Q$ is:

$$
P\left(w \mid M_{Q}\right)=\frac{Q(w)}{|Q|}
$$

where $Q(w)$ is the count of word $w$ in the query $Q$ and $|Q|=\sum_{w} Q(w)$ is the query's length.

We created our inverse collection frequency weighted query models as follows:

$$
P\left(w \mid M_{Q}\right)=-\frac{1}{Z} Q(w) \log P(w \mid C)
$$

where $P(w \mid C)$ is the MLE model of the collection, and $Z$ is the normalization factor so that the query model sums to 1. Taking the negative of the $\log ,-\log P(w \mid C)$, gives an amount proportional to the inverse collection frequency.

This ICF weighting approach is analogous to the inverse document frequency and may seem ad-hoc in construction. Our goal with the ICF weighted query is to provide a means to test our hypothesis. Our goal is not to propose a new way to estimate a query model. We hope that further work might result in a compelling formal integration of the idea into language modeling retrieval.

\section{EXPERIMENTS}

We compared the inverse collection frequency query models created via equation 1 to the MLE query models. For our queries, we used the title and description fields of the TREC topics 301-450, which are the ad-hoc topics for TREC 
Title Queries

\begin{tabular}{lcrrc} 
Test Set & MLE & ICF & \% Imp. & Sig. Level \\
\hline TREC 6 & 0.228 & 0.234 & $2.6 \%$ & 0.06 \\
TREC 7 & 0.182 & 0.189 & $3.9 \%$ & 0.12 \\
TREC 8 & 0.247 & 0.246 & $-0.3 \%$ & 0.81 \\
All: 6,7,8 & 0.219 & 0.223 & $1.9 \%$ & 0.04 \\
\multicolumn{5}{c}{} \\
Test Set & MLE & ICF & \% Imp. & Sig. Level \\
\hline TREC 6 & 0.195 & 0.212 & $8.9 \%$ & $<0.01$ \\
TREC 7 & 0.183 & 0.194 & $6.4 \%$ & 0.03 \\
TREC 8 & 0.228 & 0.238 & $4.4 \%$ & 0.05 \\
All: 6,7,8 & 0.202 & 0.215 & $6.4 \%$ & $<0.01$
\end{tabular}

Table 1: Mean average precision results for title and description queries. MLE is the maximum likelihood query. ICF is the query model constructed using the inverse collection frequency as per equation 1.

6,7 , and 8 . The collection was TREC volumes 4 and 5 minus the Congressional Record. We used the Lemur retrieval toolkit for our experiments. We stemmed all words with the Krovetz stemmer and used an in-house stopword list of 418 words. We ranked documents by their cross entropy with the query.

We used three-fold cross validation to set the document smoothing parameters. Each set of TREC topics was a fold. For example, the TREC 7 and 8 topics were the training set for the TREC 6 topics. Thus our results closer represent expected performance than optimal. We did parameter sweeps of Dirichlet prior and Jelinek-Mercer smoothing. In all cases Dirichlet prior performed better. Dirichlet prior smoothing mixes the MLE document model with the MLE collection model: $P\left(w \mid M_{D}\right)=(1-\lambda) P(w \mid D)+\lambda P(w \mid C)$, where $\lambda=m /(|D|+m)$ and $m$ is the Dirichlet prior parameter.

We measured statistical significance with a paired, twosided, randomization test with 100, 000 samples.

\section{RESULTS AND DISCUSSION}

Table 1 shows the mean average precision of MLE and ICF query models for title and description queries. ICF obtained a $1.9 \%$ improvement over MLE on title queries and a $6.4 \%$ improvement on description queries. For title queries, none of the individual TREC topic sets showed statistically significant improvements. For description queries, statistically significant improvements occurred for TREC 6 and 7. ICF's performance gain comes from improving precision at recall points of 0.3 and higher.

Table 2 shows that less document smoothing is required if the retrieval method has available another way to utilize the inverse collection frequency in an IDF-like manner.

Our results show that improved retrieval performance is possible if the IDF-like role played by document smoothing is separately handled. The performance improvement may be the result of either the better use of the ICF or better document smoothing or both.

Two-stage smoothing performs well, but in the configuration tested by Zhai and Lafferty, it does not result in statistically significant performance improvements on title or description queries for TREC 7 and 8 as compared to the best

\begin{tabular}{ccc} 
& \multicolumn{2}{c}{ Dirichlet $m$} \\
Training Set & MLE & ICF \\
\hline Titles TREC 6, 7 & 800 & 500 \\
Titles TREC 6, 8 & 350 & 400 \\
Titles TREC 7, 8 & 800 & 500 \\
& & \\
Desc. TREC 6, 7 & 2500 & 1250 \\
Desc. TREC 6, 8 & 2500 & 1000 \\
Desc. TREC 7, 8 & 3000 & 1250
\end{tabular}

Table 2: The best parameter setting for Dirichlet prior smoothing on the training sets. Except for the TREC 6, 8 titles, the inverse collection frequency (ICF) query models performed better with less smoothing than the maximum likelihood estimated (MLE) models.

performance of Dirichlet prior or Jelinek-Mercer smoothing [3]. Two-stage smoothing separately models the estimation and query-modeling (IDF-like) roles of smoothing, but it still ties the IDF-like role to the amount of smoothing required. In contrast, when we separated the IDF-like role from smoothing, we obtained a modest, but statistically significant, performance improvement for description queries on TREC 7. While two-stage smoothing requires no parameter tuning, one could tune its parameters and possibly achieve similar improvements to those reported here.

\section{CONCLUSION}

Document smoothing plays several roles in language modeling retrieval [2]. One of these roles is to produce an IDFlike effect using the inverse collection frequency (ICF). We hypothesized that lightening the IDF-like responsibilities of smoothing could improve retrieval. We created ICF weighted query models and compared their performance to maximum likelihood estimated models. The ICF query models achieved a $6.4 \%$ improvement in the mean average precision while requiring less document smoothing. Language modeling retrieval may benefit from a means to incorporate an IDF-like behavior outside of document smoothing and thereby allow document smoothing to focus on its other roles.

\section{ACKNOWLEDGMENTS}

This work was supported in part by the Center for Intelligent Information Retrieval and in part by the Defense Advanced Research Projects Agency (DARPA) under contract number HR0011-06-C-0023. Any opinions, findings and conclusions or recommendations expressed in this material are those of the authors and do not necessarily reflect those of the sponsor.

\section{REFERENCES}

[1] J. M. Ponte and W. B. Croft. A language modeling approach to information retrieval. In $S I G I R$, pages 275-281, 1998.

[2] C. Zhai and J. Lafferty. A study of smoothing methods for language models applied to ad hoc information retrieval. In SIGIR, pages 334-342, 2001.

[3] C. Zhai and J. Lafferty. Two-stage language models for information retrieval. In SIGIR, pages 49-56, 2002. 